\title{
Entwicklungseinflüsse der Weltpolitik auf den europäischen Integrationsprozess
}

\author{
Werner Link*
}

Bei der ,rückblickenden Bewertung des europäischen Integrationsprozesses“ ist auffällig, dass im Mittelpunkt des Interesses die Binnenproblematik steht. ${ }^{1}$ Das war in der Integrationsliteratur von Anfang an so. Der neo-funktionalistische Interpretationsansatz, der lange Zeit vorherrschend war, blendete externe Variablen entweder ganz aus oder behandelte sie als Residualkategorien. Den sogenannten Traditionalisten (wie Alting von Geusau und Stanley Hoffmann) und den Neo-Institutionalisten (wie Joseph Nye) war es zu verdanken, dass die binnenorientierte Integrationsforschung mit der Forderung konfrontiert wurde, ,,dem externen weltpolitischen Umfeld, in dem ein Integrationsprozess stattfindet“, ,erhöhte Aufmerksamkeit“" zu schenken. ${ }^{2}$ In diesem Sinne vertrat Amitai Etzioni bereits 1965 die These, dass eine gemeinsame externe Bedrohung einen wichtigen Integrationsimpuls darstelle. ${ }^{3}$ Aber erst 1991 erschien die erste politikwissenschaftliche Monographie über die ,externen Einflüsse auf die Integration von Staaten“, in der die Autorin, Ruth Zimmerling, systematisch zwischen negativen und positiven externen zwingenden Gründen unterschied und Nyes Kategorie des externen Katalysators übernahm. ${ }^{4}$

Aus diesem Forschungsstrang stammen zwei Hypothesen, auf die die folgende Untersuchung rekurriert:

Die Hypothese über die integrationsfördernde Wirkung gemeinsamer externer Herausforderungen, und

die Hypothese über die integrationshemmende Wirkung stärkerer Bindungen einzelner Staaten an eine externe Großmacht.

\section{Analytischer Ausgangspunkt: Struktureller Realismus}

Die neo-realistische Theorie (,struktureller Realismus“) bietet die Möglichkeit, diese Hypothesen und die bisherigen Ansätze in eine systemische Theorie (d.h. in eine Theorie, die vom internationalen System ausgeht) zu integrieren. ${ }^{5}$ Die Struktur des internationalen Systems eröffnet und evoziert bestimmte Optionen und schließt andere als unrealisierbar aus. Auf gemeinsame Herausforderungen antworten die Staaten nach der Logik von Macht- und Gegenmachtbildung, entsprechend ihrer Positionierung im internationalen System. Dabei wird - entgegen wiederholter anders lautender Behauptungen - die Bedeutung der Binnen-

* Prof. Dr. Werner Link, Forschungsinstitut für Politische Wissenschaft und Europäische Fragen, Universität zu Köln.

1 Der Text basiert auf einem Vortrag, den der Verfasser auf der wissenschaftlichen Konferenz „50 Jahre Römische Verträge“" gehalten hat. Die Konferenz wurde vom Institut für Europäische Politik in Zusammenarbeit mit dem Arbeitskreis Europäische Integration und dem Centre International de Formation Européenne in Berlin durchgeführt.

2 Joseph S. Nye Jr.: Patterns and Catalysts in Regional Integration, in: Joseph S. Nye Jr. (Hrsg.): International Regionalism, Readings, Boston 1968, S. 333-349.

3 Amitai Etzioni: Political Unification, New York u.a. 1965.

4 Ruth Zimmerling: Externe Einflüsse auf die Integration von Staaten - Zur politikwissenschaftlichen Theorie regionaler Zusammenschlüsse, München 1991. Zur theoretischen Diskussion und zu empirischen Studien in den sechziger und siebziger Jahren siehe Werner Link: Die Rolle der USA im westeuropäischen Integrationsprozess, in: Aus Politik und Zeitgeschichte April/1972, S. 3-13.

5 Vgl. Werner Link: Die Entwicklungstendenzen der Europäischen Integration (EG/EU) und die neo-realistische Theorie, in: Zeitschrift für Politik 3/2001, S. 302-321. 
struktur und der internen Wirkfaktoren keineswegs geleugnet. ${ }^{6}$ Sie muss in eine Gesamtanalyse einbezogen werden. Dass ich im Folgenden auf die internen und subsystemischen Faktoren nicht eingehen werde, ist also nicht der Theorie, sondern dem mir gestellten Thema geschuldet. Demzufolge konzentriere ich mich auf die internationalen Bedingungen, die Struktur und die Prozesse des internationalen Systems, und vor allem auf deren Auswirkungen auf die europäische Integration. Und da sich das internationale System verändert hat, ist zwischen dem internationalen System vor und nach 1990 zu unterscheiden. Auf der Prozessebene ist zwischen unterschiedlichen Regulierungen des Ost-West-Konflikts zu differenzieren. ${ }^{7}$ Den Wirkungszusammenhang zwischen Weltpolitik und europäischer Integration werde ich an einigen Knotenpunkten der Entwicklung zu verdeutlichen versuchen - thesenhaft! ${ }^{8}$

\section{Im Zeichen des Ost-West-Konflikts}

Die europäische Integration begann in einer Phase, die - bezüglich der Machtverteilung im internationalen System - wie folgt charakterisiert war:

Global bestand ein Übergewicht der USA (wirtschaftlich und vor allem militärisch aufgrund des atomaren Rüstungsvorsprungs). Regional (das heißt in Europa) existierte ein konventionelles Übergewicht der Sowjetunion. Und in den europäischen Staaten herrschten wirtschaftliche und soziale Not und politische Instabilität (mit starken kommunistischen Parteien in Frankreich und Italien).

Der Ost-West-Konflikt wurde in dieser Phase konfrontativ ausgetragen. Die amerikanische Entscheidung zum ,containment " war die historische Weichenstellung. Die westeuropäischen Staaten nahmen teil an der Gegenmachtbildung unter amerikanischer Führung und waren auf die wirtschaftliche und militärische Hilfe der USA angewiesen. Die USA banden aber ihre Wirtschaftshilfe konditional an die Kooperation zwischen den westeuropäischen Staaten und an die Einbeziehung der Westzonen beziehungsweise der Bundesrepublik Deutschland. Das war die Herausforderung für die bisherige Politik Frankreichs und Großbritanniens. Während Großbritannien die integrative Weiterentwicklung der OEEC blockierte, ergriff Frankreich die Initiative für die Einleitung der westeuropäisch-kontinentalen Integration. Der Monnet-Plan war die innovative Antwort Frankreichs - die Herstellung und Sicherung des Gleichgewichts zwischen Frankreich und Deutschland durch Integration zum Zwecke der Friedenswahrung und der Verhinderung einer deutschen Hegemonie (das heißt ,,integratives Gleichgewicht“). ${ }^{9}$ Das innereuropäisch, subsystemisch begründete Integrationsprojekt war also international vermittelt. So hat man denn auch die USA als positiven ,externen Föderator" (Hans-Peter Schwarz) bezeichnet, was freilich nur mit Einschränkungen zutrifft. ${ }^{10}$ Die Sowjetunion war gewissermaßen der negative externe Föderator, und sie blieb es

6 Kenneth N. Waltz: Theory of International Politics, Reading, Mass. 1979, S. 122. Vgl. Carlo Masala: Kenneth N. Waltz, Einführung in seine Theorie und Auseinandersetzung mit seinen Kritikern, Baden-Baden 2005.

7 Siehe Werner Link: Der Ost-West-Konflikt, Stuttgart 1988.

8 Ausführlicher habe ich diese Problematik in meinem Essay „Auf dem Weg zu einem neuen Europa - Herausforderungen und Antworten“ (Baden-Baden 2006) behandelt. Dort sind auch genauere Literaturhinweise zu finden.

9 Zu diesem Typus der „balance of power“ - integratives Gleichgewicht als Gegentypus zum antagonistischen Gleichgewicht und als Steigerung des kooperativen Gleichgewichts - siehe meine früheren Beiträge u.a.: Link: Entwicklungstendenzen, 2001; Link: Ost-West-Konflikt, 1988.

10 Siehe Hans-Jürgen Schröder: Marshallplan, amerikanische Deutschlandpolitik und europäische Integration 1947-1950, in: Aus Politik und Zeitgeschichte B 22/1987, S. 3-17. Schröder kommt zu dem Schluss, dass die amerikanische Regierung den Integrationsgedanken wegen der ,special relationship“ zu Großbritannien ,,nicht konsequent“ gefördert hat: „Möglicherweise hat aber gerade diese Ambivalenz der amerikanischen Politik der europäischen Integration insofern ungewollt einen Integrationsschub gegeben, weil sie die deutsch-französische Verständigung provoziert hat." (S. 16). 
in der ganzen Ära des Ost-West-Konflikts und der Bipolarität. Konrad Adenauer schrieb in seinen Erinnerungen: ${ }^{11}$,Sowjetrussland wurde immer wieder für die europäischen Einigungsbestrebungen zu einem Bundesgenossen wider Willen.“

In der ersten Phase des Ost-West-Konflikts war - unter den genannten Bedingungen - im Sicherheits- und Verteidigungsbereich eine unabhängige und eigenständige westeuropäische Gegenmachtbildung nicht problemadäquat und strukturell unrealistisch. Sie stand im Widerspruch zur notwendigen gemeinsamen transatlantischen Verteidigungsorganisation unter amerikanischer Hegemonie, wie sie 1949 mit der NATO auch tatsächlich geschaffen worden war. ${ }^{12}$ In dem Moment, in dem bei den Verhandlungen über die Europäische Verteidigungsgemeinschaft (EVG) die konsequente Unterordnung der EVG unter die NATO vorgenommen war und im Krisenfall der Bundesrepublik Deutschland ein Platz am NATO-Tisch eingeräumt wurde, war der derart grundlegend geänderte Pleven-Plan für Frankreich uninteressant.

Die Organisation der Sicherheit der westeuropäischen Staaten im atlantischen Rahmen hatte jedoch den positiven Effekt, dass das europäische Integrationsprojekt von der sicherheitspolitischen Aufgabe entlastet und so die ökonomische Integration erleichtert wurde. Die innereuropäischen, subsystemischen Gründe, die zur Europäischen Wirtschaftsgemeinschaft (EWG) führten, können als bekannt vorausgesetzt werden. Nicht übersehen werden sollte, dass auch das EWG-Projekt unter dem Einfluss der weltpolitischen Entwicklung zustande kam. Das Scheitern der Genfer Konferenz (1955) und die Doppelkrise in Ungarn und am Suez-Kanal (Oktober 1956) waren nachweisbar wichtige Katalysatoren. ${ }^{13}$

Die europäische Integration war zugleich auch die Antwort der westeuropäischen Staaten auf das Übergewicht der USA innerhalb des westlichen Teilsystems. Auch das wird häufig übersehen! Europa sollte durch Integration allmählich zu einer ,force d'équilibre“ weltweit und im Verhältnis zur westlichen Führungsmacht werden ${ }^{14}$ - im Sinne einer kooperativen Balance, nicht im Sinne einer antagonistischen Beziehung. Diese Intention, die Monnet in seinem Gründungsmemorandum vom 5. Mai 1950 klar formulierte und die Adenauer am 9. Mai 1950 öffentlich artikulierte, ${ }^{15}$ zieht sich wie ein roter Faden durch die Entwicklungsgeschichte der europäischen Integration.

Realpolitisch konnte sie unter den Bedingungen des Ost-West-Konflikts zunächst nur im ökonomischen Bereich wirksam werden - als Antwort auf den ökonomischen ,défi americain“. ${ }^{16}$ Die wirtschafts- und handelspolitische Herausforderung der USA wurde in den sechziger Jahren zum externen Impuls für den Versuch, den Integrationsprozess im Sinne einer Vereinheitlichung der Willensbildung und der Außenaktionen weiterzutreiben. Diese Versuche waren erfolgreich: Bei den GATT-Verhandlungen der Dillon- und KennedyRunde (1961/62 und 1964-1967) war es der Faktor USA, der die EWG-Länder zu einem gemeinsamen Auftreten bewog. ${ }^{17}$

11 Konrad Adenauer: Erinnerungen 1955-1959, Stuttgart 1967, S. 252.

12 Der hegemoniale Anspruch wurde von der amerikanischen Regierung von Anfang an deutlich formuliert; siehe die Besprechung mit den europäischen Verbündeten am Vorabend der Unterzeichnung des NATO-Vertrags (Memorandum of Conversation, The White House, 3. April 1949, abgedruckt in: Vierteljahrshefte für Zeitgeschichte 3/1992, S. 415-423).

13 Siehe u.a. die Zeugnisse von Konrad Adenauer: Erinnerungen, 1967, S. 223 ff. und S. 273; und Alfred MüllerArmack: Auf dem Weg nach Europa, Stuttgart 1971, S. 73 und S. 123.

14 Jean Monnet: Le Mémorandum du 3 Mai 1950, abgedruckt in: Politique Étrangère 1/1993, S. 121-125.

15 Ebenda und Bundeskanzler Adenauer am 9. Mai 1950, zitiert in: Die Welt, 10. Mai 1950.

16 Jean-Jacques Servan-Schreiber: Die amerikanische Herausforderung, Hamburg 1970.

17 David Coombes: Politics and Bureaucracy in the European Community, London 1970; Werner Feld: The European Common Market and the World, Engelwood Cliffs/New York 1967. 
Hingegen hatte de Gaulles Versuch, die politische Integration über den Fouchet-Plan und dann über den deutsch-französischen Vertrag (1963) in deutlicher Abgrenzung gegenüber dem amerikanischen Einfluss einzuleiten, keine Erfolgschancen - vor allem wegen der engen existenziellen Bindung der Bundesrepublik Deutschland an die amerikanische nukleare Schutzmacht. Die USA instrumentalisierten diese Abhängigkeit erfolgreich auf diplomatischer und zwischengesellschaftlicher Ebene. ${ }^{18}$ Deutschland wurde auf atlantischem Kurs gehalten, nicht jedoch Frankreich. Der Versuch, Frankreich zur ,atlantischen Unterordnung“ zu zwingen, war - wie Couve de Murville in seinem Memoiren schrieb ${ }^{19}$ - in dieser Zeit das Haupthindernis für eine politische Weiterentwicklung der europäischen Integration.

Die Entwicklungseinflüsse der Weltpolitik waren Ende der sechziger und Anfang der siebziger Jahre noch stärker als zuvor, weil sich die Machtverteilung im internationalen System und die Regulierung des Ost-West-Konflikts gravierend änderten. Einige Stichworte mögen genügen: Erlangung der nuklearen Zweitschlagfähigkeit der Sowjetunion und infolgedessen Beginn der, era of negotiations' zwischen den antagonistischen Supermächten (,détente'); Zusammenbruch des Währungssystems von Bretton Woods; Verbindung des Ost-West-Konflikts mit dem eskalierenden Nahost-Konflikt (Oktober-Krieg von 1973, ÖlBoykott der OPEC).

Die Entwicklung der Europäischen Gemeinschaft in dieser Phase lässt sich mit folgenden Stichworten skizzieren: Haager Gipfelkonferenz (Dezember 1969) mit der Entscheidung über den EG-Beitritt Großbritanniens, Irlands und Dänemarks; Gründung der Europäischen Politischen Zusammenarbeit (EPZ) und nachfolgende Erklärung über die europäische Identität (Kopenhagen, 14. Dezember 1973); Einleitung des Euro-Arabischen Dialogs; Gymnicher Gentlemen's Agreement über das Verhältnis zwischen der Europäischen Gemeinschaft und den USA (1974).

Analysiert man diese Entwicklung der europäischen Integration, so ist sie klar als Antwort auf die genannten externen Herausforderungen zu erkennen. Die Quellenbelege sind zahlreich. Schon die zeitgenössischen öffentlichen Erklärungen sprechen eine deutliche Sprache. So bekundeten zum Beispiel die EWG-Kommissare Mansholt und Dahrendorf am 24. Oktober 1971 vor der Presse in Washington, dass die am 15. August unilateral von der amerikanischen Regierung eingeleitete Politik (Beendigung des Systems von Bretton Woods und Verhängung einer Surcharge auf Importe) ,,die Europäische Gemeinschaft zu einer größeren Einheit“" gebracht habe. ${ }^{20}$ Und in der Identitätserklärung der EG/EPZ (14. Dezember 1973) hieß es: ${ }^{21}$ „Die in der Welt eingetretenen Veränderungen und die wachsende Zusammenballung von Macht und Verantwortung in den Händen ganz weniger Weltmächte verlangen, dass Europa sich zusammenschließt“, um „die ihm zukommende Rolle“ zu spielen - als ,ein Element des Gleichgewichts und ein Pol der Zusammenarbeit mit allen Nationen."

Nach der Veröffentlichung der damals geheimen diplomatischen Akten ist noch detaillierter als zuvor nachweisbar, wie die erwähnten weltpolitischen Ereignisse und Entscheidungen - insbesondere das amerikanisch-sowjetische Anti-Atomkriegsabkommen und Kissingers Versuch, durch eine neue atlantische Erklärung Westeuropa zu disziplinieren - den

18 Zur diplomatischen Ebene siehe: Akten zur Auswärtigen Politik der Bundesrepublik Deutschland (AAPD), 1963, München 1994; zur zwischengesellschaftlichen Ebene siehe Werner Link: Deutsche und amerikanische Gewerkschaften und Geschäftsleute 1945-1975, Düsseldorf 1978, S. 147 und S. 231-232.

19 Maurice Couve de Murville: Une politique étrangère, Paris 1971.

20 Zitiert nach Süddeutsche Zeitung, 25.10.1971.

21 Auswärtiges Amt (Hrsg.): Europäische Politische Zusammenarbeit (EPZ), Dokumentation, Bonn 1984, Dokument 5, S. 50-55, hier S. 52, Hervorhebung hinzugefügt. 
europäischen Integrationsprozess positiv beeinflusst haben. So war der französische Entwurf für die Identitätserklärung bestimmt von der Einsicht, dass Frankreich aus eigener Kraft und auf sich gestellt den Auswirkungen der amerikanisch-sowjetischen Annäherung auf Europa nicht Paroli bieten könne. Deshalb musste die EPZ gestärkt werden. Ihre Ratio war, ,gegenüber (den) westlichen und östlichen Kraftzentren, besonders wenn beide zusammenwirkten“, die Europäische Gemeinschaft politisch handlungsfähig zu machen. ${ }^{22}$ Wider Willen wurde die Kissinger-Initiative für eine neue Atlantik-Charta, die ohne Konsultation mit den westeuropäischen Regierungen eingeleitet worden war, zum externen Katalysator; sie gab dem europäischen Einigungsprozess ,neue politische Impulse“. ${ }^{23}$ Und es klingt geradezu wie eine neo-realistische Einschätzung, wenn Bundeskanzler Brandt dem italienischen Ministerpräsidenten Rumor am 8. November 1973 erklärte: ${ }^{24}$ „Aus der Weltstruktur ergebe sich, dass wir mit Phasen rechnen müssten, in denen das Verhältnis zwischen den beiden Supermächten besser sei als das zwischen den Vereinigten Staaten und Europa; je gemeinsamer Westeuropa auftrete, um so besser könne es derartige Phasen bestehen.“

Bemerkenswert ist schließlich, dass die Anstöße für regelmäßige EG-Gipfeltreffen (für den späteren Europäischen Rat) von dem Nahost-Konflikt und der Ölkrise verstärkt wurden. Sie wirkten also auch institutionell als externe Katalysatoren der europäischen Integration.

In der zweiten Hälfte der siebziger Jahre setzte sich die Tendenz der ,Europäisierung ‘ unter dem Einfluss der währungspolitischen Turbulenzen fort. Die Schaffung des Europäischen Währungssystems (1978) war die Antwort auf die amerikanische Währungspolitik, um eine „europäische Gegenposition“ (Bundeskanzler Schmidt) aufzubauen.25 Das „Wall Street Journal“ (5. Dezember 1978) lag nicht ganz falsch mit der Meinung, ,dass Deutschland und Frankreich in Wahrheit daran interessiert seien, eine neue Supermacht zu schmieden, die von der amerikanischen Politik und Wirtschaft unabhängig sein werde“". In der Politik gegenüber der Dritten Welt reagierte die Europäische Gemeinschaft auf die Herausforderung einer Neuen Weltwirtschaftsordnung mit der Intensivierung der Zusammenarbeit mit den ehemaligen Kolonien (Staaten Afrikas, der Karibik und des Pazifiks, AKP). Das EG-AKP-Abkommen von Lomé (1975/76) dokumentierte die eigenständige Entwicklungsländer-Politik der Europäischen Gemeinschaft - trotz der Einwände der USA gegen derartige Präferenzbeziehungen und im Kontrast zur universellen Politik der United Nations Conference on Trade and Development (UNCTAD).

In den achtziger Jahren, in denen zunächst die neuerliche konfrontative Regulierung des Ost-West-Konflikts und dann die neue umfassende Détente weltpolitisch bestimmend waren, sind die Auswirkungen der Weltpolitik auf den europäischen Integrationsprozess nach ähnlichem Muster manifest geworden wie in den entsprechenden vorausgegangenen Phasen. Auf dem Höhepunkt der neuen Ost-West-Konfrontation hatte die amerikanische Strategische Verteidigungsinitiative (SDI), die Präsident Reagan am 23. März 1983 ankündigte, einerseits die Spaltung der Europäischen Gemeinschaft zur Folge; sie war aber andererseits auch der Anstoß für eine gemeinsame europäische Technologiepolitik (Eureka). In der Phase der umfassenden Détente eröffnete sich die Möglichkeit, unter günstigen weltpolitischen Bedingungen den europäischen Integrationsprozess weiterzuentwickeln (Beitritt Spa-

22 AAPD, 1973, III, Dok. 317.

23 Runderlass des AA vom 18. Dezember 1973, in: AAPD, 1973, III, Dok. 422.

24 AAPD, 1973, III, Dok. 365 (Hervorhebung hinzugefügt).

25 Bundeskanzler Schmidt in der Sitzung des Parteivorstands der SPD am 27.2.1978; siehe Werner Link: Außenund Deutschlandpolitik in der Ära Schmidt 1974-1982, in: Geschichte der Bundesrepublik Deutschland, Bd. 5/ II, Stuttgart/Mannheim 1987, S. 287. 
niens und Portugals, Unterzeichnung der Einheitlichen Europäischen Akte, Schengener Abkommen und Delors-Plan zur Vollendung des Binnenmarkts und zur Währungsunion).

Als Zwischenresümee lässt sich Folgendes zusammenfassen:

In der Ära der Bipolarität und des Ost-West-Konflikts wurden die Einflüsse der Weltpolitik auf die Entstehung und Entwicklung der europäischen Integration vermittelt durch die Politik der beiden antagonistischen Supermächte und durch das jeweilige Verhältnis zwischen ihnen, also durch die Regulierungsmodi des Ost-West-Konflikts. Solange der OstWest-Konflikt bestimmend war, war die gemeinsame Herausforderung durch die Sowjetunion sowohl für die Entwicklung der europäischen Integration als auch für das Verhältnis zwischen Europa und den USA dominant. Die gemeinsame Herausforderung wirkte auf den europäischen Integrationsprozess einerseits in der Regel fördernd ein. Andererseits bewirkte sie die sicherheits- und verteidigungspolitische Beschränkung der europäischen Integration und die Abschwächung der politischen Balance-Tendenz gegenüber den USA. Die Konkretisierung dieser Tendenz war unter den Bedingungen der konfrontativen Regulierung des Ost-West-Konflikts schwieriger als in den Détente-Phasen. Spezielle Abhängigkeiten (insbesondere der Bundesrepublik Deutschland) und spezielle Bindungen (,special relationships ${ }^{\circ}$ ) einzelner Staaten spielten eine hemmende Rolle. Aber wirksam war diese BalanceTendenz durchgängig. Selbstbehauptung Europas in der Welt - das war das Leitmotiv.

\section{Neue Herausforderungen der Weltpolitik}

Der Zusammenbruch des alten internationalen Systems und die neuen internationalen Strukturen und Prozesse (die hier als bekannt vorausgesetzt werden) haben die Gründung und Entwicklung der Europäischen Union in einer Weise beeinflusst, die teils als Fortsetzung und Steigerung der Tendenzen der vorausgegangenen Epoche, teils als neue Antworten auf neue weltpolitische Herausforderungen zu interpretieren ist. Auch dazu können an dieser Stelle nur einige Hinweise gegeben werden.

Die Schaffung der Europäischen Währungsunion - als Fortsetzung des Europäischen Währungssystems - stand in der Tradition der Gegenmachtbildung zum US-Dollar (neben dem internen Hauptmotiv, Deutschland beziehungsweise die D-Mark europäisch einzubinden). ${ }^{26}$ Die spezielle Sterling-Dollar-Beziehung, an der Großbritannien festhalten wollte, und andere Sonderheiten führten zur währungspolitischen ,differenzierten Integration“ (Euro-Gruppe nach festen Beitrittskriterien).

,Maastricht" wurde nur zu einem integrationspolitischem Teilerfolg, weil unter anderem Großbritannien anti-föderal und atlantisch orientiert war und in der Sicherheitspolitik die NATO-Priorität (mit der Bindung an die USA) hemmend wirkte. Und der gesamteuropäische Konföderationsplan Mitterrands scheiterte an den mittel- und osteuropäischen Staaten, die den Beitritt zur Europäischen Union und zur NATO (und damit die Erlangung des Schutzes der USA) wünschten. Immerhin: die Umwandlung der EPZ in die Gemeinsame Außenund Sicherheitspolitik (GASP) und dann die allmähliche Ausweitung des europäischen Integrationsprozesses auf die Verteidigungspolitik wurden möglich und wirklich, weil mit der Auflösung des Warschauer Paktes und dem Untergang der Sowjetunion der Grund für das strukturelle Nebeneinander und für das daraus resultierende Spannungsverhältnis zwischen der atlantischen Organisation der europäischen Sicherheit und der europäischen Organisation der wirtschaftlichen und politischen Zusammenarbeit der europäischen Staaten , objek-

26 In Frankreich wurde deutlicher als in Deutschland ausgesprochen, dass der Euro ein Gegengewicht zum USDollar werden sollte - ,um die amerikanische Hegemonie zu bekämpfen“ (Chirac), siehe Link: Entwicklungstendenzen, 2001, S. 308. 
tiv` entfallen war. Die duale Struktur und das Spannungsverhältnis wurden allerdings durch die Osterweiterung der NATO und durch den Sieg der NATO im ,institutionellen Wettbewerb“ (Kori Schake) ${ }^{27}$ reproduziert und durch die Bestrebungen eines Teils der EU-Staaten, nun eine eigenständige Verteidigungsstruktur gegen amerikanischen Widerstand zu schaffen, verschärft. In diesem Prozess wurden die USA vom einstigen , externen Föderator ' zum externen Divisor - bis hin zur jüngsten Sonderbeziehung der USA zu Polen und Tschechien bei der amerikanischen Raketenabwehr. Zugleich haben aber die USA - wider Willen - im , alten`Europa die Integrationstendenzen gestärkt.

Die Erfahrungen der Abhängigkeit Europas von den USA im Verlauf der Balkan-Konflikte hat als Katalysator für die Europäische Sicherheits- und Verteidigungspolitik gewirkt. Von den entsprechenden Beschlüssen des Kölner EU-Gipfels im Jahre 1999 bis hin zum Aufbau der Battle-groups (an dem 18 der 25 beziehungsweise 27 EU-Staaten teilnehmen) und schließlich bei der Gründung der Europäischen Verteidigungsagentur ist diese katalysatorische Wirkung des Faktors USA feststellbar, teilweise hemmend, teilweise - wider Willen - stimulierend. Schon zuvor (1991/92) hatte die scharfe amerikanische Demarche gegen eine europäische Verteidigungsorganisation ${ }^{28}$ die Gründung der Eurokorps nicht verhindern können, aber bewirkt, dass der Teilnehmerkreis beschränkt blieb.

Im Irak-Konflikt - der seine ordnungspolitische Bedeutung wegen der erstmaligen Anwendung der imperialen Nationalen Sicherheitsstrategie der USA (2002) erhielt - ist es den USA gelungen, die Europäische Union sichtbar zu spalten: Der von den USA initiierte proamerikanische öffentliche Brief der Acht vom 30. Januar 2003 wurde von fünf der fünfzehn EU-Mitglieder und von drei mittel-osteuropäischen Beitrittskandidaten unterzeichnet - ohne Konsultation der anderen EU-Staaten und zu einem Zeitpunkt, als die EU-Ratspräsidentschaft dabei war, eine einheitliche Linie zu erarbeiten. Die von James Kurth prognostizierte „Dialektik von imperialer Expansion und Opposition“29 produzierte also nicht den einheitlichen Akteur Europäische Union, sondern dynamisierte den Prozess der Differenzierung. Zugleich entstand jedoch aus der Opposition einer Avantgarde-Gruppe (Vierer-Gipfel in Tervuren, 29. April 2003) eine Bewegung zur Stärkung der Europäischen Sicherheits- und Verteidigungspolitik - mit der Zwischenstation des deutsch-französisch-britischen Gipfels in Berlin (20. September 2003) und der Entschließung der Gesamt-EU auf der Außenministerkonferenz von Neapel (19. November 2003) sowie dem Protokoll 23 des Verfassungsvertrags, dessen Inhalt inzwischen umgesetzt worden ist (Verteidigungsagentur, Battle-groups), und zwar nach Maßgabe der differenzierten Integration mit festen Kriterien wie bei der EWU.

Als Reaktion auf den Iran-Konflikt ist integrationspolitisch ein neuer, zukunftsweisender Aktionstypus entwickelt worden: Auftragsverhandlungen durch die drei europäischen Führungsmächte Deutschland, Frankreich und Großbritannien (EU-3) nebst dem Hohen Vertreter für die GASP. Inhaltlich und taktisch unterscheidet sich die EU-Diplomatie deutlich von der amerikanischen Politik, nämlich kooperative Konfliktregulierung statt konfrontativer Konfliktregulierung (mit der Hintergrunddrohung der militärischen Intervention und des erzwungenen Regimewechsels). Ob die Eigenständigkeit der EU-Politik nach dem Einschwenken auf die Sanktionspolitik der USA erhalten bleiben wird, ist freilich eine offene Frage. ${ }^{30}$

27 Vgl. Werner Link: Die NATO im Geflecht internationaler Organisationen, in: Aus Politik und Zeitgeschichte B 11/1999, S. 9-18.

28 Die (nicht veröffentlichte) amerikanische Demarche datiert vom 21. Februar 1991 (Kopie im Besitz des Verfassers); zum Wortlaut siehe Link: Die NATO, 1999, S. 15-16.

29 James Kurth: Confronting the Unipolar Moment, in: Current History 101/2002, S. 403-408. Vgl. auch Stephen M. Walt: Taming American Power - The Global Response to U.S. Primacy, New York/London 2005.

30 Siehe dazu Link: Auf dem Weg zu einem neuen Europa, 2006, S. 83 ff. 
Der transnationale islamistische Terrorismus, der durch den Angriff auf die USA am 11. September 2001 und die folgende „Kriegserklärung“ des amerikanischen Präsidenten eine Zäsur in der Weltpolitik erzeugt hat, wirkt sich widersprüchlich auf den europäischen Integrationsprozess aus. Die neuartige Herausforderung, die alle zivilisierten Staaten (ihre Sicherheit und ihr innerstaatliches Gewaltmonopol) betrifft, hat gemeinsame und unterschiedliche Antworten hervorgerufen.

In Afghanistan beteiligten beziehungsweise beteiligen sich die EU-Staaten je einzeln (sowie auch außereuropäische Staaten) an der amerikanischen Operation Enduring Freedom. An der Stabilisierung Afghanistans (ISAF) haben die europäischen Staaten ebenfalls zunächst mit nationalen Kontinenten teilgenommen, dann den Rahmen der NATO gewählt. Was die weltweite Dimension anbelangt, so hat indes die transnationale islamistische Herausforderung integrationspolitisch vereinheitlichend gewirkt. Die Europäische Union war in der Lage, sich konzeptionell auf eine gemeinsame Europäische Sicherheitsstrategie (2003) zu einigen, die sich von der Nationalen Sicherheitsstrategie der USA (2002) klar unterscheidet. ${ }^{31}$ Und innereuropäisch hat die terroristische Bedrohung den Kooperations- und Integrationsprozess gefördert (Zusammenarbeit im Polizei- und Justizbereich).

Vor allem aber hat die säkulare ökonomische Tendenz der Globalisierung die Entwicklung der Europäischen Union in mannigfacher Weise angetrieben. Diejenigen Staaten, die im vergangenen Jahrzehnt der Europäischen Union beigetreten sind, taten dies nach eigenem Bekunden nicht zuletzt deshalb, weil sie nur so im Globalisierungsprozess bestehen zu können glaubten. ${ }^{32}$ Die europäischen Staats- und Regierungschefs haben wiederholt die Fortsetzung des Integrationsprozesses mit der Globalisierung begründet: „Europe puissance“ als notwendige Antwort auf die Globalisierung. ${ }^{33}$ Die gemeinsamen Wettbewerbsanstrengungen, die die Europäische Union 2000 in Konkurrenz zu den USA beschlossen haben (Lissabon-Strategie), sind der sichtbare Ausdruck dieser integrationspolitischen Ratio. Generell stärkt die Globalisierung die Regionalisierungstendenz weltweit, und das regionale Integrationsprojekt Europäische Union profitiert davon in spezieller Weise. ${ }^{34}$

Schließlich ist zu beobachten, dass neue globale Herausforderungen, die mit der ökonomischen Globalisierung indirekt zusammenhängen, dazu geführt haben, dass der Integrationsprozess auf mindestens drei neue Politikbereiche ausgeweitet wurde. Der Migrationsstrom, der aus Ländern und Regionen stammt, die - wie Afrika - eher die Verlierer der Globalisierung sind, hat die Europäische Union zu einer gemeinsamen Regulierungspolitik veranlasst. Die energiepolitische externe Abhängigkeit - insbesondere von Russland - hat Ansätze einer gemeinsamen EU-Energiepolitik evoziert und im Verhältnis zu Russland die Tendenz für eine kooperative Balance-Politik (, strategische Partnerschaft ${ }^{\circ}$ ) intensiviert. Dabei zeigt sich interessanterweise, dass die unterschiedliche Positionierung einzelner EUStaaten (wie zum Beispiel Polens) die europäische Einheitlichkeit hemmt und dass Russland als externe Großmacht - ähnlich wie die USA in anderen Politikbereichen - bilaterale Vereinbarungen mit einzelnen EU-Staaten (wie zum Beispiel mit Deutschland) bevorzugt. Auf die globale Herausforderung des Klimawandels hat die Europäische Union mit einer gemeinsamen Klimapolitik geantwortet und weltweit eine Vorreiterrolle übernommen, und zwar inhaltlich in Opposition zur Politik der USA. Bei der Verabschiedung und Durchfüh-

31 Ebenda, S. 81-82 und S. 90-91.

32 Vgl. Werner Link: Die Neuordnung der Weltpolitik, München 2001, Kap. IV und S. 156.

33 So u.a. Bundeskanzler Schröder in seiner Rede vor der französischen Nationalversammlung am 30. November 1999, abgedruckt in: Internationale Politik 4/2000, S. $94-99$ (insbesondere S. 97).

34 Ausführlich dazu Link: Die Neuordnung der Weltpolitik, 2001, Kap. IV, 4 und 5. 
rung des Kyoto-Protokolls und bei den Vereinbarungen des G8-Gipfels in Heiligendamm hat sich diese Gemeinsamkeit bewährt.

\section{Gesamteinschätzung}

Neben den innereuropäischen Herausforderungen (die hier nicht erörtert werden konnten) waren und sind es die gemeinsamen strukturellen und prozesshaften Herausforderungen der internationalen Politik, auf die die europäischen Staaten mit dem europäischen Integrationsprojekt geantwortet haben beziehungsweise antworten, und zwar im Sinne der Friedenswahrung und der Selbstbehauptung Europas, um als eigenständiges Kraftzentrum zu einem kooperativen Gleichgewichtsfaktor und Pol in einer pluralistischen, multipolaren Welt zu werden. Die externen, weltpolitischen Einflüsse auf den europäischen Integrationsprozess sind in beiden Epochen - vor und nach 1990 - in vielfältiger Weise eindeutig nachweisbar. Was die Wirkungen anbelangt, so bestätigt die empirische Analyse die beiden eingangs genannten Hypothesen und die These über externe Katalysatoren.

Die unterschiedliche internationale Positionierung der einzelnen $\mathrm{E}(\mathrm{W}) \mathrm{G} / \mathrm{EU}-$ Staaten und speziellen Bindungen einzelner E(W)G/EU-Staaten an externe Großmächte (hauptsächlich an die USA) erklären, dass die weltpolitischen Einflüsse im alten internationalen System (1949/50-1990/91) oftmals den Integrationsprozess gehemmt und im neuen internationalen System (nach 1990/91) eine differenzierte Integration gefördert haben. Indem in der erweiterten EU-27 die Heterogenität größer als zuvor ist und die vereinheitlichende Wirkung der gemeinsamen Bedrohung durch die Sowjetunion nicht mehr existiert, sind die Wirkungen der externen Einflüsse differenzierender als zuvor. Der organisatorische Modus der daraus folgenden differenzierten Integration heißt in der EU-Terminologie „Verstärkte“ beziehungsweise „Ständige Strukturierte Zusammenarbeit“. Sie ist in der beschriebenen Weise durch externe Wirkfaktoren bedingt - und selbstredend auch durch interne Faktoren. In einer Gesamtanalyse wird man über die Gewichtung zwischen externen und internen Einflüssen streiten können, und man sollte dies sinnvollerweise phasen- und fallspezifisch tun.

\section{EU-Strategie für den Südkaukasus}

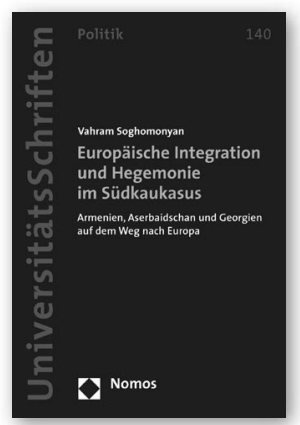

\section{Europäische Integration und Hegemonie im} Südkaukasus

Armenien, Aserbaidschan und Georgien auf dem Weg nach Europa

Von Vahram Soghomonyan

2007, 204 S., brosch., 46,-€, ISBN 978-3-8329-2403-4

(Nomos Universitätsschriften - Politik, Bd. 140)

Bitte bestellen Sie bei Ihrer Buchhandlung oder bei Nomos | Telefon 07221/2104-37 | Fax -43 www.nomos.de | sabine.horn@nomos.de

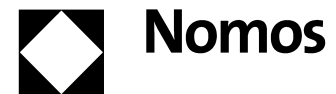

